\title{
Onshore and Offshore Wind Speed Distributions at the Western Waters in Taiwan
}

\author{
Jui-Fang Tsai, Hsien-Kuo Chang, Jin-Cheng Liou, and Lian-Sheng Ho
}

\begin{abstract}
Wind speed data for 2010 at eight stations located in three coastal areas of Taiwan were used to fit three kinds of Weilbull distributions in this study. Bimodal Gamma-Weibull distribution is examined the best among three distributions for all wind speed data comparing three tests for goodness-of-fit. Key indicators for a bimodal distribution are the weighting parameter approaching 0.5 , variation of peak speeds of monthly distribution and peak speed ratio. Topographical effect of high central mountains of Taiwan on offshore wind speeds at southern Taiwan is stronger than at northern Taiwan. It explains that the distribution of offshore wind speeds is bimodal at northern Taiwan and unimodal at southern Taiwan.
\end{abstract}

Index Terms-Offshore/onshore wind speed, bimodal Weibull distribution, goodness-of-fit.

\section{INTRODUCTION}

On March 11, 2011, a 9.0 earthquake shocked the northeastern coast of Japan and a tsunami following the earthquake caused the failure of a nuclear power plant as well as a massive loss of life and property. Therefore, alternative power sources instead of nuclear power are again investigated worldwide. One of several alternative power sources is wind power that can provide a steady supply of electricity with clean and sustainable use. Suitable wind farms in Taiwan were evaluated and many wind turbine generators were established in coastal areas in the recent ten years. In the future planning, some offshore wind turbine generators will be built in the Taiwan Strait. It is important to understand the characteristics of onshore and offshore in Taiwan waters for future development of wind power.

Furthermore understanding and modeling wind speed statistics is a key for a better understanding of atmospheric turbulence and diffusion and for use in such practical applications as air quality and pollution transport modeling, estimation of wind loads on buildings, prediction of atmospheric or space probe and missile trajectory and wind power analysis.

Wind speed is a key factor for designing wind power stations. Statistical analysis on wind speed data around a wind field is commonly used to study their characteristics, providing important references for wind energy and

Manuscript received December 19, 2015; revised April 7, 2016. This work was supported in part by the Harbor and Marine Technology Center in Taiwan under Grant MOTC-IOT-103-H2EB001f .

Jui-Fang Tsai, Hsien-Kuo Chang, and Jin-Cheng Liou are with Department of Civil Engineering, National Chiao Tung University, Hsinchu 300, Taiwan (e-mail: juifang0321@hotmail.com, hkc@mail.nctu.edu.tw, jcliou.cv87g@nctu.edu.tw).

Lian-Sheng Ho is with Harbor and Marine Technology Center, Taichung435, Taiwan (e-mail: hls@mail.ihmt.gov.tw). engineering design. Different probability density distribution of wind speeds has been investigated to show wind power for the development of wind farms [1]-[7]. The Weibull distribution is an important distribution especially for reliability and maintainability analysis.

Both linear least square method and maximum likelihood method for estimating two Weibull parameters were analyzed to select locations of installing wind turbine generators [8]. The most accurate and efficient in six numerical methods was examined to ascertain how closely the measured data follow the two-parameter Weibull PDF [9]. Wind energy conversion characteristics of Hatiya Island in Bangladesh were studied using statistical fitting Weibull distribution for wind speed data [10]. Wind speed data at three different heights for the whole year of 2008 at Thasala, southern Thailand were analyzed using two-parameter Weibull distribution [11]. The validity domain of the Weibull distribution for wind statistics was assessed and an alternative expression of more fitted wind speed distribution was suggested [12].

The Weibull distribution is commonly used for wind speed data. However, single peak of such a distribution is no more suitable for two-peak distributed wind speed data. Bimodal Weibull distribution for wind speeds is examined to be the best among 14 commonly and previously used probability density functions for fitting a set of data [13].

The characteristics of offshore winds in Taiwanese waters have been clarified using in situ coastal wind data for decades. Three kinds of distributions, i.e. unimodal Weibull, bimodal Weibull and Gamma-Weibull distributions, are selected to fit the offshore/onshore wind speeds at eight stations in the western waters of Taiwan [14]-[16]. T The occurrence probability and intensity of diurnal and semidiurnal winds over Taiwanese waters were estimated using wavelet-based rotary spectral analysis and significance level theory [17].

In order to understand the difference between onshore and offshore wind speeds for establishment of applicable wind power stations some Weilbul-like distributions are proposed for fitting the wind speed data at different stations instead of single sample in the western waters, Taiwan. he best fitting of wind speeds among three chosen distributions are determined by three statistical measures for goodness-of-fit R-Square, root mean square error and the Kolmogorov-Smirnov test. The difference between distributions of annual wind speeds resulting from monthly variation of wind speeds was indicated. The results can be used as a reference of onshore and offshore wind energy potentials inthe application to designing wind power station in Taiwan.

\section{BACKGROUND AND DATA}


Wind speed data at eight stations measured by the Harbor and Marine Technology Center, Institute of Transportation, Ministry of Transportation and Communications in Taiwan were used in this study. These stations are located at the Taipei harbor in northern Taiwan, the Taichung harbor in central Taiwan and the Anping harbor in southern Taiwan. These locations are shown in Fig. 1. Detailed locations and names of onshore/offshore stations in each area are shown in Fig. 2.

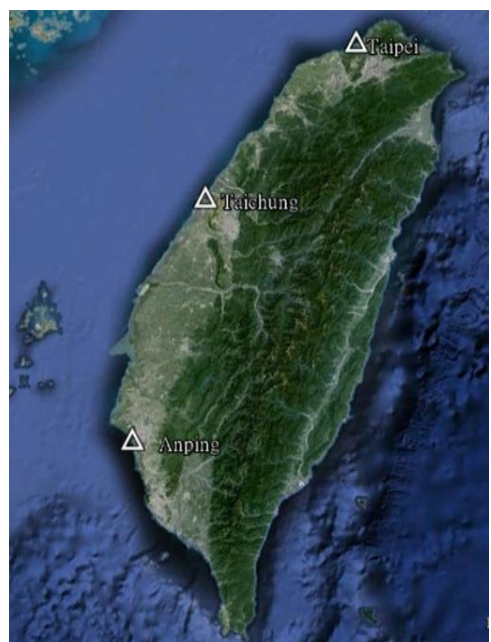

Fig. 1. Locations of the Taipe, the Taichung and the Anping harbors.

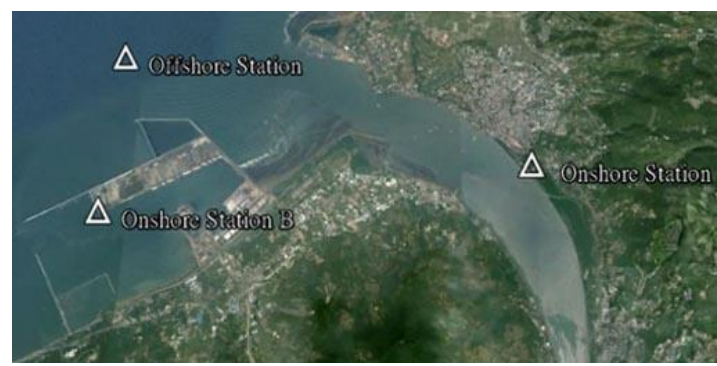

(a) The Taipei harbor

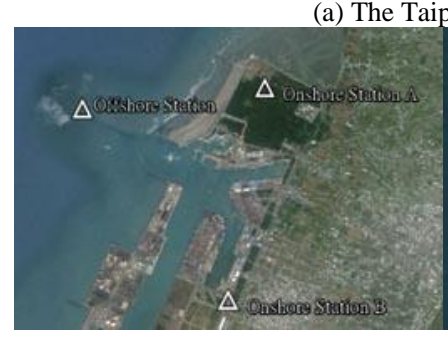

(b) The Taichung harbor

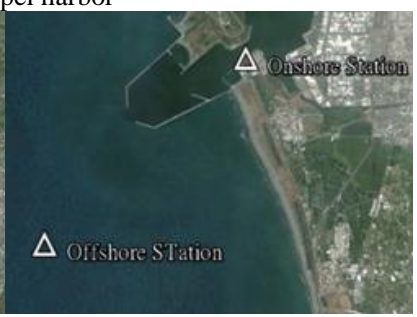

(c) The Anping harbor
Fig. 2. Locations of offshore and onshore stations at three harbors of Taiwan.

The wind speeds are measured by Young Brand marine wind monitor (model 50106). The wind speed sensor is a four blade helicoid propeller. Propeller rotation produces an AC sine wave voltage signal with frequency directly proportional to wind speed. The wind direction sensor is a rugged yet lightweight vane with a sufficiently low aspect ratio to assure good fidelity in fluctuating wind conditions. Accuracy of wind monitor is $\pm 0.3 \mathrm{~m} / \mathrm{s}$ or $1 \%$ for speed and \pm 3 degrees for wind direction. Signal output is at a frequency of $1 \mathrm{~Hz}$. Mean velocity is averaged from all data of instant velocity over every ten minutes. Hourly data of mean velocity at o'clock sharp for the whole year are used. Data are transmitted and collected from the sensor transducer to ground data-base station through $3 \mathrm{G}$ mobile telecommunications every ten minutes.
Sometimes data in one year are unavailable due to data transmission or device failure. Except that unavailable data occur in February at the onshore station of the Taichung harbor all data at other stations are complete and available for the whole 2010. Therefore, considering the availability, and completeness the whole hourly data at eight stations were used for 2010 in the study.

\section{Probability Density FunCtion AND MODEL PERFORMANCE}

Weibull, bimodal Weibull and bimodal Gamma-Weibull distributions, denoted by $\mathrm{W}, \mathrm{WW}$ and GW, respectively, are selected for data fitting. WW is examined to be valid for wind speeds by Morgan et al. [13] and GW is proposed by Chang et al. [15]. The probability density function (PDF) and corresponding cumulate probability function $(\mathrm{CDF})$ are defined as follows:

Weibull distribution:

$$
\begin{gathered}
f(U ; \alpha, \beta)=\frac{\beta U^{\beta-1}}{\alpha^{\beta}} \exp \left(-\left(\frac{U}{\alpha}\right)^{B}\right) \\
F(U ; \alpha, \beta)=1-\exp \left(-\left(\frac{U}{\alpha}\right)^{B}\right)
\end{gathered}
$$

where $\alpha$ is the scale parameter and $\beta$ is the shape parameter.

Bimodal Weibull distribution:

$$
\begin{aligned}
f\left(U ; \alpha_{1}, \beta_{1}, \alpha_{2}, \beta_{2}, \omega\right)= & \omega \frac{\beta_{1} U^{\beta_{1}-1}}{\alpha_{1}^{\beta_{1}}} \exp \left[-\left(\frac{U}{\alpha_{1}}\right)^{\beta_{1}}\right] \\
& +(1-\omega) \frac{\beta_{2} U^{\beta_{2}-1}}{\alpha_{2}^{\beta_{2}}} \exp \left[-\left(\frac{U}{\alpha_{2}}\right)^{\beta_{2}}\right] \\
F\left(U ; \alpha_{1}, \beta_{1}, \alpha_{2}, \beta_{2}, \omega\right) & =\omega\left(1-\exp \left[-\left(\frac{U}{\alpha_{1}}\right)^{\beta_{1}}\right]\right) \\
& +(1-\omega)\left(1-\exp \left[-\left(\frac{U}{\alpha_{2}}\right)^{\beta_{2}}\right]\right)
\end{aligned}
$$

where the subscripts 1 and 2 indicate the parameters in the primary part and the second part, respectively, and $\omega$ is the weighting for the primary part.

Gamma-Weibull distribution:

$$
\begin{aligned}
f\left(U ; k, \theta, \alpha_{3}, \beta_{3}, \omega\right) & =\omega \frac{U^{k-1}}{\theta^{k} \Gamma(k)} \exp \left[-\frac{U}{\theta}\right] \\
& +(1-\omega) \frac{\beta_{3} U^{\beta_{3}-1}}{\alpha_{3}^{\beta 3}} \exp \left[-\left(\frac{U}{\alpha_{3}}\right)^{\beta_{3}}\right] \\
F\left(U ; k, \theta, \alpha_{3}, \beta_{3}, \omega\right)= & \omega\left(\frac{1}{\Gamma(k)} \int_{0}^{\frac{U}{\theta}}\left(\frac{t}{\theta}\right)^{k-1} \exp \left[-\frac{U}{\theta}\right] d\left(\frac{t}{k}\right)\right) \\
& +(1-\omega)\left(1-\exp \left[-\left(\frac{U}{\alpha_{3}}\right)^{\beta_{3}}\right]\right)
\end{aligned}
$$

where $\Gamma(k)$ is the gamma function evaluated at $k$, indicating the shape parameter, and $\theta$ is the scale parameter. 
Three indexes of model performance, the coefficient of determination, the Kolmogorov-Smirnov test and the root mean square error are commonly chosen to examine the goodness of fit of an observed distribution to a theoretical one. The statistical meaning and definition of these indexes are given as follows.

The coefficient of determination, $R^{2}$, shows the ratio of the variance of the predictions to the sample variance and is defined as

$$
R^{2}=1-\frac{\sum_{i=1}^{N}\left(Y_{i}-y_{i}\right)^{2}}{\sum_{i=1}^{N}\left(Y_{i}-\bar{Y}\right)^{2}}
$$

where $Y_{i}$ and $y_{i}$ are the measurements and predictions, respectively. $R^{2}$ ranges from 0 and $1 . R^{2}=1$ indicates that the regression line perfectly fits the data, while $R^{2}=0$ indicates that the measurements and predictions are independent.

Kolmogorov-Smirnov test (K-S test) is a nonparametric test of the equality of a continuous probability distribution, $F(U)$, that can be used to compare a sample with a reference or empirical probability distribution, $E(U)$, which is obtained by $N$ ordered data points. The Kolmogorov-Smirnov test statistic is defined as

$$
D=\max _{1<i<N}\left(F(U)-\frac{i-1}{N}, \frac{i}{N}-F(U)\right)
$$

For fitting one data set, a probability distribution having a smaller $D$ than other distributions indicates that the distribution is more suitable for fitting the data with an empirical probability distribution than others.

Root mean square error (RMSE) represents the sample standard deviation of the differences between predicted values and observed values. RMSE is a good measure of accuracy, but only to compare forecasting errors of different models for a particular variable and not between variables. It is defined

$$
R M S E=\sqrt{\frac{\sum_{i=1}^{N}(Y i-y i)^{2}}{N}}
$$

\section{RESULTS AND DISCUSSION}

\section{A. Assessment for Goodness of Fit}

In order to assess the goodness of fit of all observed distributions to three chosen distributions the three statistical measures were computed for eight cases and list in Table I. For each case the value of each row in the bold-faced type indicates the best goodness of fit to such distribution rather than other two distributions according to such an index. When a distribution has the most bold-faced values for each case, the distribution is examined to be the best goodness of fit among three chosen distributions.

For example, for the case of the offshore station of the Taipei harbor, three bold-faced values in the first panel of
Table I are list in the last column. The reason is that GW has smaller values of K-S and RMSE and higher $R^{2}$ than other two distributions for such case. Therefore, it is concluded that GW is a better distribution for fitting wind speeds at the offshore station of the Taipei harbor than W and WW. For the case of onshore station A of the Taipei harbor, WW has one bold-faced K-S value and GW has other two bold-faced indexes in the second panel. However, the differences of both values between WW and GW are tiny. Both WW and GW with equivalent data fitting suitably describe an empirical probability distribution for such data. Three bold-faced indexes are all list in the second column in the third panel. The result indicates that the best data fitting for the distribution is WW for the case of the onshore station B of the Taipei harbor.

For the Taichung harbor, all best indexes list in the second column of the second panel show that distributed data at the offshore station can be better fitted by WW rather than by W and GW. Best indexes being in the third column indicate that the distribution of wind speeds can be suitably fitted by GW at the onshore station A. At the onshore station B, WW and GW are equivalently valid for the distribution of wind speeds.

For the Anping harbor, all indexes are list in the last panel of Table I. The distribution of wind speeds at offshore and onshore stations can be more validly fitted by GW than by $\mathrm{W}$ and WW. W is the worst one to fit the distribution of wind speeds among three distributions for all data sets. From the above discussion WW is suggested for the distribution of wind speeds at onshore stations B of the Taipei harbor and for those at offshore stations of the Taichung harbor. For other stations GW is suggested for the distribution of wind speeds.

\begin{tabular}{|c|c|c|c|c|c|}
\hline Harbor & Station & Index & W & בWW & $\overline{\mathrm{GW}}$ \\
\hline \multirow{9}{*}{ Taipei } & \multirow{3}{*}{ Offshore } & $\mathrm{R}^{2}$ & 0.9023 & 0.9865 & 0.9968 \\
\hline & & K-S & 0.0535 & 0.0182 & 0.0099 \\
\hline & & RMSE & 0.0122 & 0.0044 & 0.0022 \\
\hline & \multirow{3}{*}{ On- A } & $\mathrm{R}^{2}$ & 0.9848 & 0.9963 & 0.9968 \\
\hline & & $\mathrm{K}-\mathrm{S}$ & 0.0243 & 0.0191 & 0.0201 \\
\hline & & RMSE & 0.0163 & 0.0076 & 0.0069 \\
\hline & \multirow{3}{*}{ On-B } & $\mathrm{R}^{2}$ & 0.9981 & 0.9999 & 0.9999 \\
\hline & & $\mathrm{K}-\mathrm{S}$ & 0.0304 & 0.0086 & 0.0093 \\
\hline & & RMSE & 0.0073 & 0.0027 & 0.0030 \\
\hline \multirow{9}{*}{$\begin{array}{c}\text { Taichun } \\
\mathrm{g}\end{array}$} & \multirow{3}{*}{ Offshore } & $\mathrm{R}^{2}$ & 0.9807 & 0.9905 & 0.9845 \\
\hline & & $\mathrm{K}-\mathrm{S}$ & 0.0300 & 0.0162 & 0.0201 \\
\hline & & RMSE & 0.0040 & 0.0029 & 0.0037 \\
\hline & \multirow{3}{*}{ On- A } & $\mathrm{R}^{2}$ & 0.9076 & 0.9862 & 0.9892 \\
\hline & & K-S & 0.0583 & 0.0183 & 0.0174 \\
\hline & & RMSE & 0.0158 & 0.0064 & 0.0060 \\
\hline & \multirow{3}{*}{ On-B } & $\mathrm{R}^{2}$ & 0.9981 & 0.9981 & 0.9982 \\
\hline & & K-S & 0.0086 & 0.0086 & 0.0098 \\
\hline & & RMSE & 0.0024 & 0.0025 & 0.0024 \\
\hline \multirow{6}{*}{ Anping } & \multirow{4}{*}{ Offshore } & $\mathrm{R}^{2}$ & 0.9853 & 0.9919 & 0.9950 \\
\hline & & K-S & 0.0226 & 0.0230 & 0.0091 \\
\hline & & RMSE & 0.0062 & 0.0044 & 0.0035 \\
\hline & & $\mathrm{R}^{2}$ & 0.9967 & 0.9882 & 0.9958 \\
\hline & \multirow[t]{2}{*}{ Onshore } & K-S & 0.0336 & 0.0243 & 0.0085 \\
\hline & & RMSE & 0.0124 & 0.0075 & 0.0044 \\
\hline
\end{tabular}

TABLE I: THREE STATISTICAL TESTS FOR THE GOODNESS OF FIT OF ALL DATA DISTRIBUTIONS TO THE W, WW AND GWD IS ATTRIBUTIONS

GW is finally chosen to investigate the data fitting in further study. The obtained values of parameters in $\mathrm{GW}$ for all data are list in Table II. Through the parameters of the Gamma and Weibull distributions associated with weighting $\omega$ in Table II, the whole distribution can be separated into the 
primary Gamma part and second Weibull part.

\begin{tabular}{|c|c|c|c|c|c|c|}
\hline Harbor & Station & $K$ & $\theta$ & $\alpha_{3}$ & $\beta_{3}$ & $\omega$ \\
\hline \multirow{3}{*}{ Taipei } & Offshore & 2.3753 & 1.9914 & 11.5498 & 4.6610 & 0.6715 \\
\hline & On-A & 1.4440 & 0.6643 & 2.5479 & 1.9631 & 0.2414 \\
\hline & On-B & 1.9360 & 2.2122 & 9.2845 & 3.2171 & 0.6337 \\
\hline \multirow{3}{*}{ Taichung } & Offshore & 2.2062 & 3.2224 & 17.4267 & 5.0551 & 0.8280 \\
\hline & On-A & 3.5338 & 0.8274 & 9.8099 & 2.4680 & 0.6233 \\
\hline & On-B & 2.1384 & 1.8855 & 5.0762 & 1.7826 & 0.1485 \\
\hline \multirow[t]{2}{*}{ Anping } & Offshore & 1.2993 & 4.3519 & 6.2077 & 2.2337 & 0.1908 \\
\hline & Onshore & $\begin{array}{c}12.683 \\
6\end{array}$ & 0.3301 & 4.1838 & 1.6000 & 0.3321 \\
\hline
\end{tabular}

\section{B. Components of Wind Speed Distribution}

The fitted PDF and CDF are plotted in the top figure (a) from Fig. 3 to Fig. 10 for each station. The corresponding primary and secondary components of GW are plotted in the bottom figure (b).

For three stations at the Taipei harbor Fig. 3(a) and Fig. 4(a) show that the offshore wind speeds are ranged from $0 \mathrm{~m} / \mathrm{s}$ and $20 \mathrm{~m} / \mathrm{s}$ with a wide bandwidth and onshore wind speeds ranged from $0 \mathrm{~m} / \mathrm{s}$ to $7 \mathrm{~m} / \mathrm{s}$ with a narrow bandwidth. The comparison between these two figures illustrates stronger wind speeds at the offshore station than those at the onshore station A. Fig. 3(b) shows two peaks of the primary and second components located at $2.63 \mathrm{~m} / \mathrm{s}$ and $10.96 \mathrm{~m} / \mathrm{s}$, whose frequencies are $10.79 \%$ and $5.00 \%$, respectively.

As shown in Fig. 4(b), two peaks of two components occur at $0.34 \mathrm{~m} / \mathrm{s}$ and $1.75 \mathrm{~m} / \mathrm{s}$, respectively, whose frequencies are $18.25 \%$ and $25.23 \%$. Larger difference between two peak speeds of two components in Fig. 3(b) than that between Fig. 4(b) demonstrates that the distribution at the offshore station displays bimodal and the distribution at the onshore station $\mathrm{A}$ looks approximately unimodal.

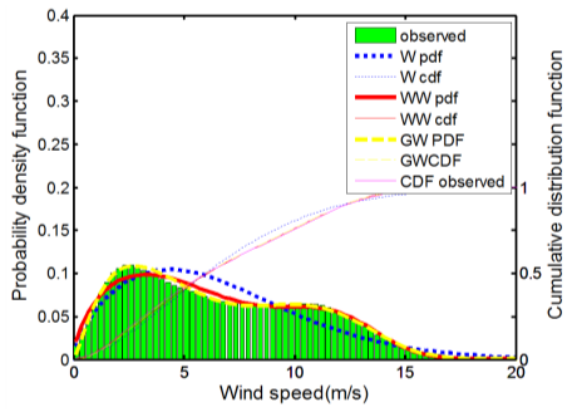

(a) the whole pdf and cdf

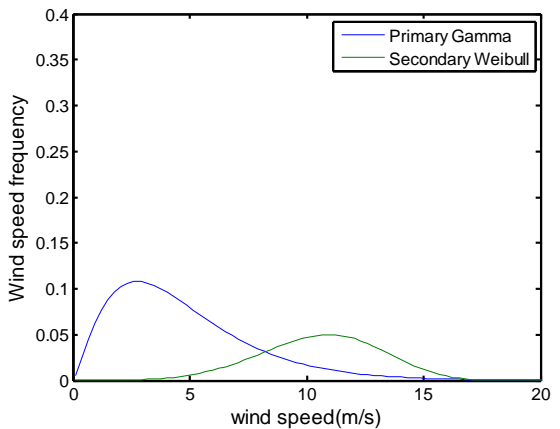

(b) primary Gamma and secondary Weibull distributions

Fig. 3. The whole distribution and corresponding primary and second components of wind speeds at the offshore station of the Taipei harbor.

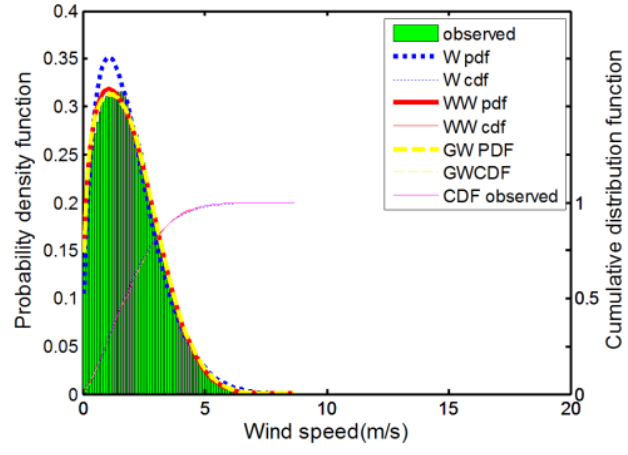

(a) the whole pdf and cdf

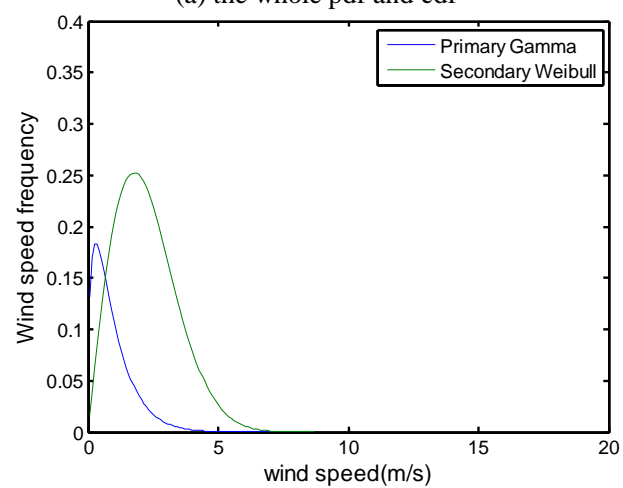

(b) primary Gamma and secondary Weibull distributions

Fig. 4. Caption as Fig. 3 for the onshore station A of Taipei harbor.

Fig. 5(a) displays a long right-hand tailed distribution with single peak of which the wind speeds are between $0 \mathrm{~m} / \mathrm{s}$ and $17.9 \mathrm{~m} / \mathrm{s}$ for onshore station B. However, Fig. 5(b) shows two different peaks whose peak speedsare $2.18 \mathrm{~m} / \mathrm{s}$ and $8.38 \mathrm{~m} / \mathrm{s}$ and frequenciesare $10.82 \%$ and $4.92 \%$, respectively. Fig. 5(b) with two significantly separated components looks like Fig. 3(b)

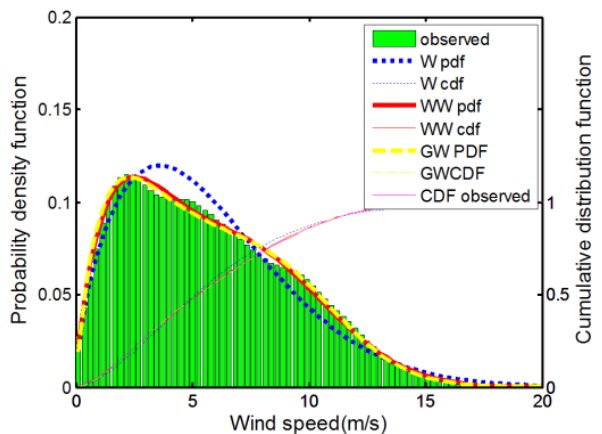

(a) the whole pdf and cdf

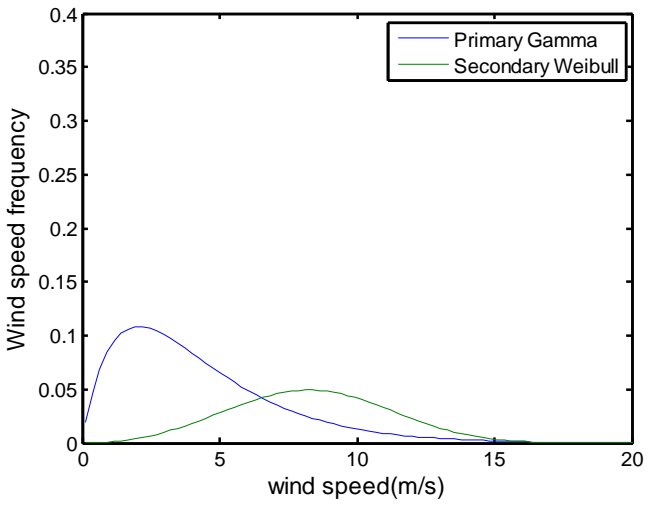

(b) primary Gamma and secondary Weibull distributions

Fig. 5. Caption as Fig. 3 for the onshore station B of the Taipei harbor

The peak speed ratio is defined as the ratio of the peak speed of each component between two stations. The peak 
frequency ratio is the ratio of the peak frequency of each component between two stations. Two peak speed ratios between the offshore station and the onshore station A are7.73 and 6.26, respectively. The peak frequency ratios are 0.59 and 0.19 , respectively. Two peak speed ratios and corresponding peak frequency ratios between the offshore station and the onshore station B are 1.20 and 1.30, respectively, and 0.99 and 1.01 , respectively.

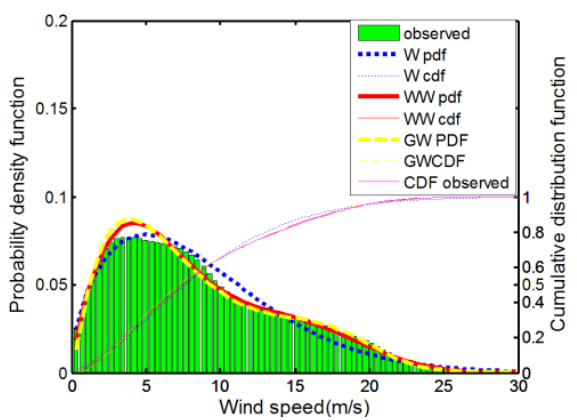

(a) the whole pdf and cdf

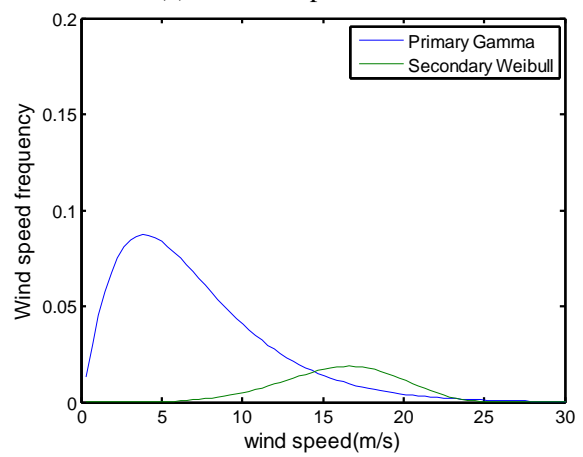

(b) primary Gamma and secondary Weibull distributions

Fig. 6. Caption as Fig. 3 for the offshore station of the Taichung harbor.

Three weightings of GW in Table I are $0.6715,0.2414$ and 0.6337 , respectively. When the weighting of a binomial distribution is close to 1 or zero, the primary component is significantly larger or smaller than the other second component. The whole distribution has a single peak. The weighting close to 0.5 implies that both components are equivalent and the whole distribution displays bimodal. The weightings of the offshore and the onshore station $\mathrm{B}$ approaching 0.6 explain why both distributions are bimodal.

By two components of a bimodal distribution and peak speed ratio as well as peak frequency ration it is easy to explain the reason that the difference between distributions of the offshore station and of the onshore station A at the Taipei harbor is large, but small between and the offshore station and of the onshore station B. The onshore station A is $5 \mathrm{~km}$ away from the coast and near northeastern hill. In the winter monsoons are strong winds blowing northeast wards. Because of topographical sheltering reduced wind speeds at the onshore station A are measured. On the other hand, onshore station B is located at the end of northern breakwaters of the Taipei harbor. There are no obstacles to change the offshore winds, and the wind speed and probability density distribution are similar to those of the offshore station.

For three stations at the Taichung harbor, Fig. 6(a), Fig. 7(a) and Fig. 8(a) show that the offshore wind speeds are ranged from $0 \mathrm{~m} / \mathrm{s}$ and $25.60 \mathrm{~m} / \mathrm{s}$ and onshore wind speeds ranged from $0 \mathrm{~m} / \mathrm{s}$ to $19.50 \mathrm{~m} / \mathrm{s}$, and $0 \mathrm{~m} / \mathrm{s}$ to $14.94 \mathrm{~m} / \mathrm{s}$, respectively, for station $\mathrm{A}$ and $\mathrm{B}$. Three fitted probability density functions appear unimodal and right-hand tailed, special at the offshore station and the onshore station B.

Three weightings of GW in Table I are $0.8280,0.6233$ and 0.1485 , respectively. The weightings of the offshore and the onshore station B deviating from 0.5 implying that both distributions should seem unimodal.

Fig. 6(b) shows two peaks of the primary and second components located at $3.82 \mathrm{~m} / \mathrm{s}$ and $16.64 \mathrm{~m} / \mathrm{s}$, whose frequencies are $8.72 \%$ and $1.87 \%$, respectively. As shown in Fig. 7(b), two peaks of two components occur at $2.08 \mathrm{~m} / \mathrm{s}$ and $7.88 \mathrm{~m} / \mathrm{s}$, respectively, whose frequencies are $18.27 \%$ and $3.83 \%$. Fig. 8(b) shows two peaks of the primary and second components located at $2.23 \mathrm{~m} / \mathrm{s}$ and $3.29 \mathrm{~m} / \mathrm{s}$, whose frequencies are $2.73 \%$ and $13.42 \%$, respectively.

Two peak speed ratios between the offshore station and the onshore station A are 1.83 and 2.11, respectively. The corresponding peak frequency ratios are 0.47 and 0.48 , respectively. Two peak speed ratios and corresponding peak frequency ratios between the offshore station and the onshore station B are 1.71 and 5.05, respectively, and 3.19 and 0.13 , respectively.

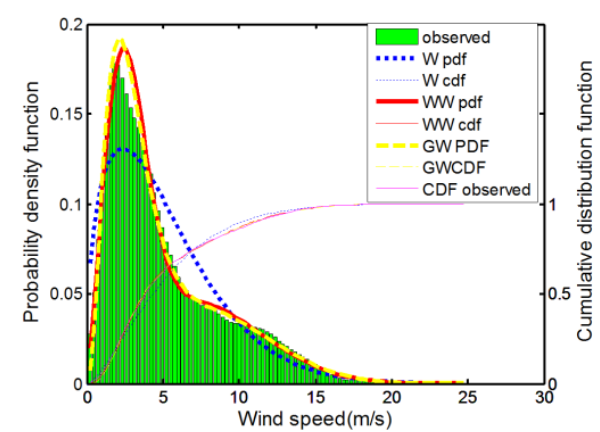

(a) the whole pdf and cdf

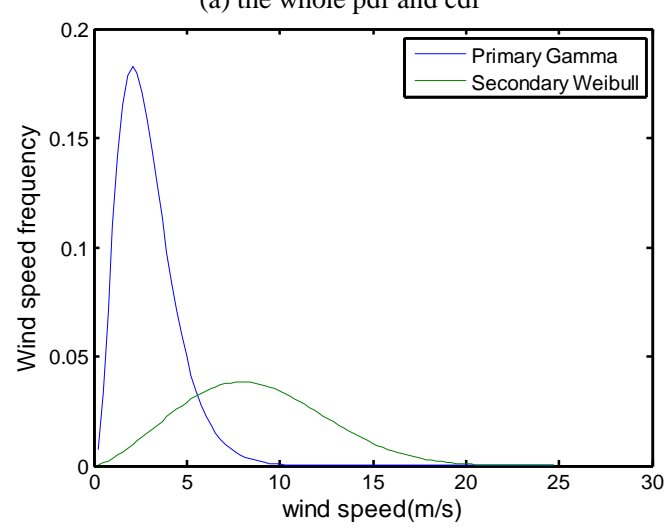

(b) primary Gamma and secondary Weibull distributions Fig. 7. Caption as Fig. 3 for the onshore station A of the Taichung harbor.

For two stations at the Anping harbor, Fig. 9(a) and Fig. 10 (a) indicate that the offshore wind speeds are ranged from 0 $\mathrm{m} / \mathrm{s}$ and $18 \mathrm{~m} / \mathrm{s}$ and onshore wind speeds ranged from $0 \mathrm{~m} / \mathrm{s}$ to $12.8 \mathrm{~m} / \mathrm{s}$. Two weightings in the third panel in Table I are 0.1908 and 0.3321 , respectively. The weighting of the offshore approaching zero shows the distribution unimodal. The other distribution at the offshore should appear insignificantly bimodal.

Fig. 9(b) shows two peaks of the primary and second components located at $0.87 \mathrm{~m} / \mathrm{s}$ and $4.69 \mathrm{~m} / \mathrm{s}$, whose frequencies are $2.47 \%$ and $12.07 \%$, respectively. It is shown in Fig. 10(b) that two peak speeds are $3.90 \mathrm{~m} / \mathrm{s}$ and $2.29 \mathrm{~m} / \mathrm{s}$, respectively, whose frequencies are $11.65 \%$ and $12.15 \%$. 
Two peak speed ratios between the offshore station and the onshore station are 0.22 and 2.04 , respectively. The corresponding peak frequency ratios are 0.21 and 0.99 , respectively.

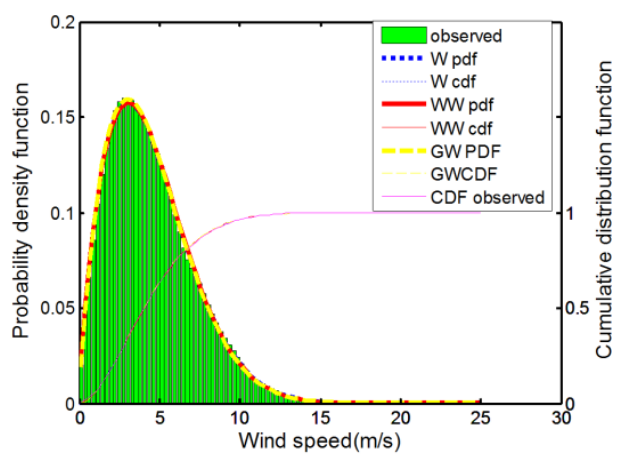

(a) the whole pdf and cdf

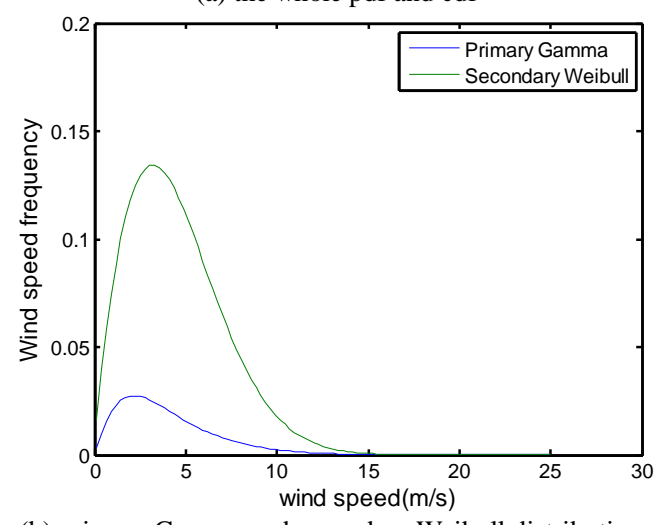

(b) primary Gamma and secondary Weibull distributions

Fig. 8. Caption as Fig. 3 for the onshore station B of the Taichung harbor.

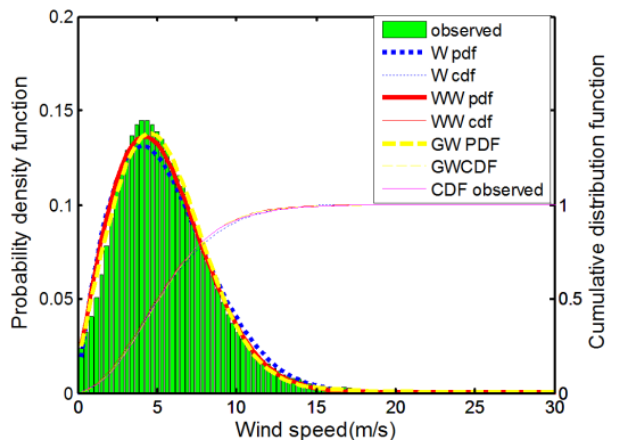

(a) the whole pdf and cdf

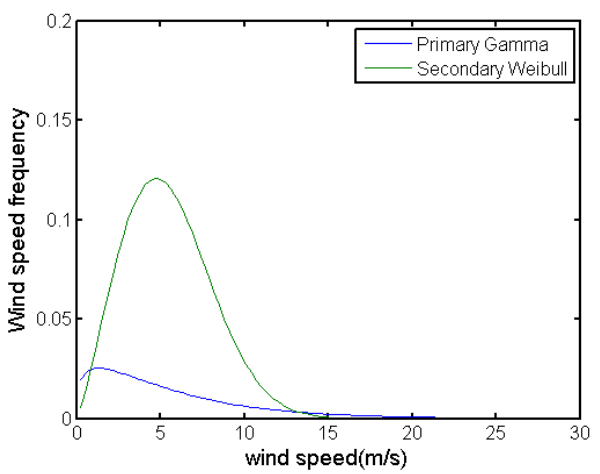

(b) primary Gamma and secondary Weibull distributions

Fig. 9. Caption as Fig. 3 for the offshore station of the Anping harbor.

Strong northeastern winds blow during the winter in Taiwan. High central mountains with the tallest peak being $3952 \mathrm{~m}$, running from the north of the island to the south in Taiwan, weaken the monsoons. Therefore, the wind speeds at the offshore station differ insignificantly from those at the onshore of the Anping harbor.

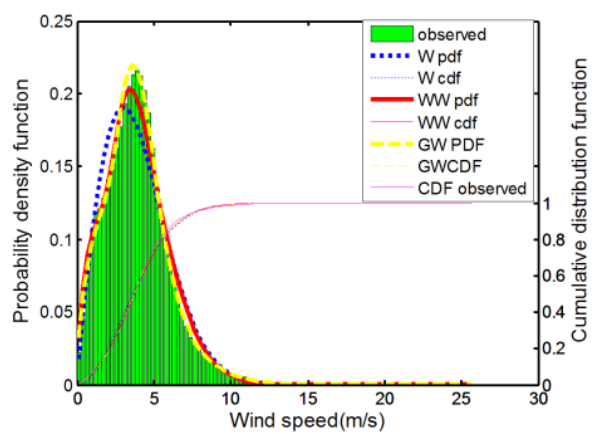

(a) the whole pdf and cdf

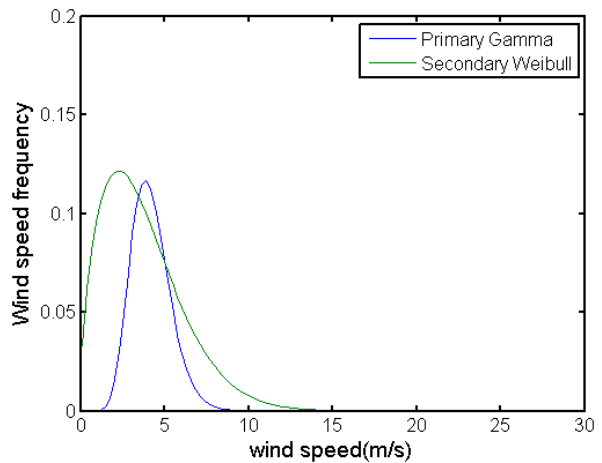

(b) primary Gamma and secondary Weibull distributions Fig. 10. Caption as Fig. 3 for the onshore station of the Anping harbor

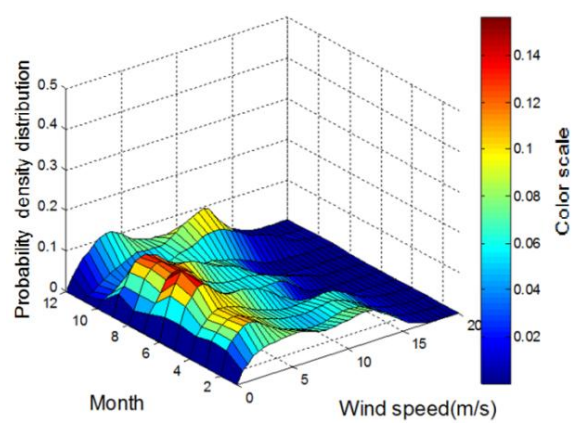

(a) surface plot

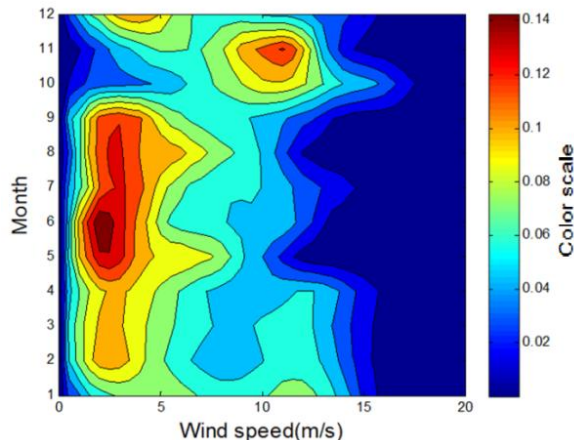

(b) contour plot

Fig. 11. Monthly distribution of wind speeds at the offshore station of the Taipei harbor.

\section{Monthly Distribution of Wind Speeds}

In the above sub-section, the weighting parameter and components of a distribution are used to demonstrate the characteristics of a bimodal wind speed distribution. However, the distribution of wind speeds is obtained from data of the whole year. We attempt to explain the reason why the two peak speeds occur. Twelve distributions of monthly wind speeds of the offshore stations at three harbors are plotted in 
surface plot and contourplot, respectively, and shown in Fig. 11 to Fig. 13.

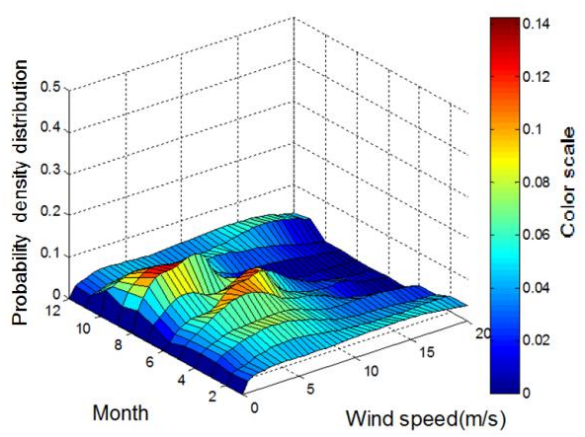

(a) surface plot

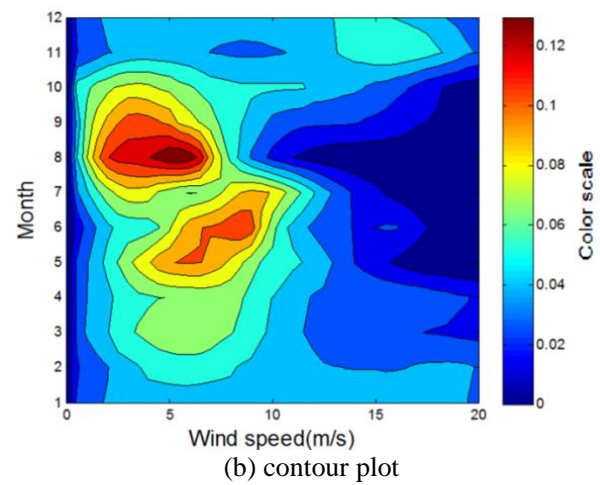

Fig. 12. Caption as Fig. 11 for the Taichung harbor.

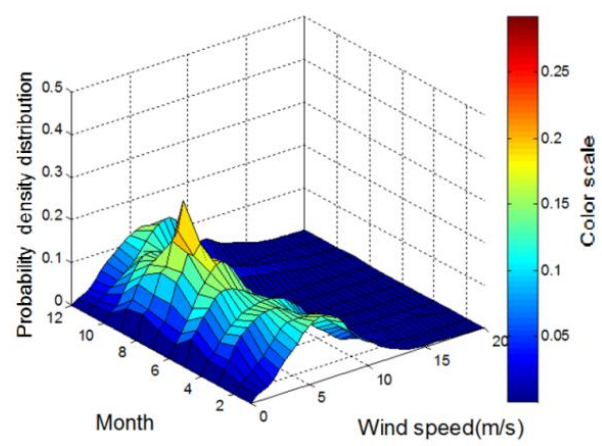

(a) surface plot

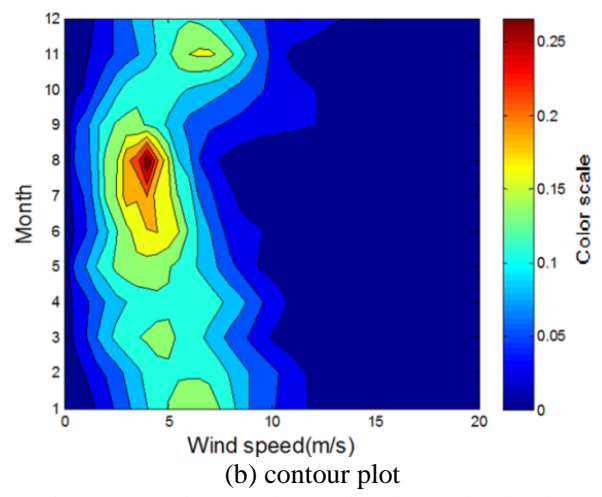

Fig. 13. Caption as Fig. 11 for theAnping harbor.

Fig. 11 indicates that monthly peak wind speeds are about $10 \mathrm{~m} / \mathrm{s}$ during winter time from September to March next year and are about $3 \mathrm{~m} / \mathrm{s}$ during the summer time from April to August. Monthly peak wind speeds in the winter significantly differ from those in the summer. Occurrence frequencies of both kinds of monthly peak velocity are approximate. Different wind speeds in the winter and in the summer result in a bimodal distribution of the whole wind speeds.

For the Taichung harbor Fig. 12 shows that monthly peak wind speeds vary in a range of $3 \mathrm{~m} / \mathrm{s}$ to $8 \mathrm{~m} / \mathrm{s}$ during the periods from February to November and about $13 \mathrm{~m} / \mathrm{s}$ for January and December. Due to various monthly peaks over long time the distribution of the whole wind speeds with a long right-land tail is wide but non-obviously bimodal.

Twelve peak wind speeds of the monthly distribution, shown in Fig. 13, of the Anping harbor vary from $3 \mathrm{~m} / \mathrm{s}$ to6 $\mathrm{m} / \mathrm{s}$. The result indicates the distribution of the whole wind speeds having a single peak and being narrow banded.

\section{CONCLUSION}

Data of wind speeds at eight onshore or offshore stations at the western waters in Taiwan for the whole year were fitted by single peak and bimodal distributions. Some conclusions are made and list below:

1) Three tests for goodness-of-fit of three chosen distributions are examined to show that the bimodal Gamma-Weibull is most valid for most data sets.

2) The weighting parameter in the bimodal Gamma-Weibull distribution approaching 0.5 is a key index for distinguishable peaks.

3) Ratios of two peak speeds and frequencies of the primary and the secondary components is an alternative index to explain why a distribution is bimodal.

4) Small variation of peak speeds and frequencies of monthly distribution shows single peak distribution of the whole wind speeds. Contrarily large variation of monthly peaks indicates a two-peaked distribution.

Based on the understanding of onshore and offshore wind speed data in Taiwanese western waters, the diurnal and semidiurnal oscillations in surface wind as well as the seasonal and spatial characteristics of these oscillations should be further investigated. The effects of such variations on offshore wind energy conversion are considerable and should not be ignored. The shafts of offshore wind turbines with long blades are commonly settled at a height of more than $90 \mathrm{~m}$ above the seas, the characteristics of vertically distributed wind speeds instead of those at single point are important to accurately determine energy efficiency of wind turbine in the further study.

\section{REFERENCES}

[1] F. Y. Ettoumi, H. Sauvageot, and A. E. H. Adane, "Statistical bivariate modelling of wind using first-order Markov chain and Weibull distribution," Renewable Energy, vol. 28, pp. 1787-1802, 2003.

[2] J. D. Holmes and W. W. Moriarty, "Application of the generalized Pareto distribution to extreme value analysis in wind engineering," Journal of Wind Engineering and Industrial Aerodynamics, vol. 83, pp. 1-10, 1999.

[3] O. A. Jaramillo and M. A.Borja, "Wind speed analysis in La Ventosa, Mexico: a bimodal probability distribution case," Renewable Energy, vol. 29, pp. 1613-1630, 2004.

[4] M. Li and X. Li, "MEP-type distribution function: a better alternative to Weibull function for wind speed distributions," Renewable Energy, vol. 30, pp. 1221-1240, 2005.

[5] Y. O. Xiao, Q. S. Li, Z. N. Li, and G.Q. Li, "Probability distributions of extreme wind speed and its occurrence interval," Engineering Structures, vol. 28, pp. 1173-1181, 2006.

[6] J. Zhou, E. Erdem, G. Li, and J. Shi, "Comprehensive evaluation of wind speed distribution models: A case study for North Dakota sites," Energy Conversion and Management, vol. 51, pp. 1449-1458, 2010.

[7] I. Usta and Y. M. Kantar, "Analysis of some flexible families of distributions for estimation of wind speed distributions," Applied Energy, vol. 89, pp. 355-367, 2012. 
[8] P. Bhattacharya and R. Bhattacharjee, "A study on Weibull distribution for estimating the parameters," Journal of Applied Quantitative Methods, vol. 5, pp. 234-241, 2010.

[9] D. K. Kidmo, R. Danwe, S. Y. Doka, and N. Djongyang, "Statistical analysis of wind speed distribution based on six Weibull methods for wind power evaluation in Garoua, Cameroon," Revue des Energies Renouvelables, vol. 18, pp. 105-125, 2015.

[10] A. K. Azada et al., "Analysis of wind energy conversion system using Weibulldistribution," Procedia Engineering, vol. 90, pp. 725-732, 2014 ,

[11] J. Waewsak, C. Chancham1, M. Landry, and Y. Gagnon, "An analysis of wind speed distribution at Thasala, Nakhon Si Thammarat, Thailand," Journal of Sustainable Energy \& Environment, vol. 2, pp. 51-55, 2011.

[12] P. Drobinski and C. Coulais, "Is the Weibull distribution really suited for wind statistics modeling and wind power evaluation?"

[13] E. C. Morgan, M. Lackner, R. M. Vogel, and L. G. Baise, "Probability distributions for offshore wind speeds," Energy Conversion and Management, vol. 52, pp. 15-26, 2011.

[14] T. P. Chang, "Estimation of wind energy potential using different probability density function," Applied Energy, vol. 88, pp. 1848-56, 2011.

[15] T. P. Chang, "Wind speed and power density analyses based on mixture weibull and maximum entropy distributions," International Journal of Applied Science and Engineering, vol. 1, pp. 39-46, 2010.

[16] F.J. Liu, H. H .Ko, S. S. Kuo, Y. H. Liang, and T. P. Chang, "Study on wind characteristics using bimodal mixture weibull distribution for three wind sites in Taiwan," Journal of Applied Science and Engineering, vol. 17, pp. 283-292, 2014.

[17] H. Chien, H. Y. Cheng, K. H. Yang, Y. H. Tsai, and W. T. Chang, "Diurnal and semidiurnal variability of coastal wind over Taiwanese waters," Wind Energy, vol. 18, pp. 1353-1370, 2014.

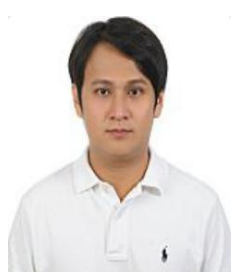

Jui-Fang Tsai was born in 1977. He is a PhD student of civil engineering in National Chiao Tung University, Taiwan. His current research interests about numerical model and statistical model of wind.

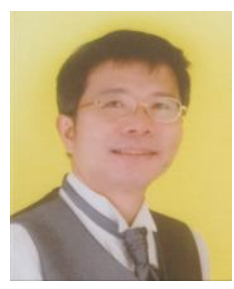

Hsien-Kuo Chang was born in 1960 . He received his $\mathrm{PhD}$ degree in hydraulic and ocean engineering from Cheng Kung University. He is currently a professor of Civil Engineering in National Chiao Tung University. He specializes in coastal engineering, remote sensing and wave mechanics.

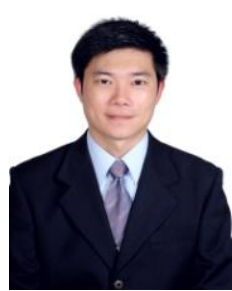

Jin-Cheng Liou was born in 1975 . He received his $\mathrm{PhD}$ degree in civil engineering from National Chiao Tung University, Taiwan, in 2005. He worked as a postdoctoral fellow at the Department of Civil Engineering in National Chiao Tung University, Taiwan. His current research interests are in oceanography numerical model and wave mechanics.

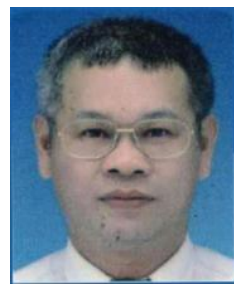

Lian-Sheng Ho received his $\mathrm{PhD}$ degree in hydraulic and ocean engineering from Cheng Kung University. $\mathrm{He}$ is currently a section chief of Harbor and Marine Technology Center, Taiwan. He specializes in coastal engineering and spectral analysis. 